\title{
ANÁLISIS SISTÉMICO DE LA SOSTENIBILIDAD ECONÓMICA DE UNIDADES DE PRODUCCIÓN AGROPECUARIA FAMILIAR EN UNA COMUNIDAD CAMPESINA DE LEBRIJA, COLOMBIA
}

\author{
SYSTEMIC ANALYSIS OF THE ECONOMIC SUSTAINABILITY OF FAMILY \\ AGRICULTURAL PRODUCTION UNITS IN A PEASANT COMMUNITY OF LEBRIJA, \\ COLOMBIA
}

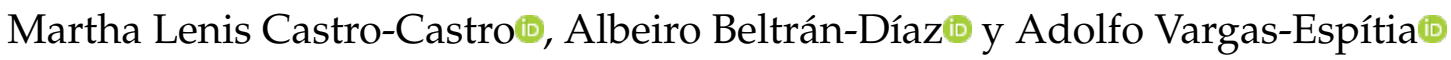

Corporación Universidad de Investigación y Desarrollo -UDI, Calle 923 55, Bucaramanga, Santander, Colombia.

*Autor para correspondencia: abeltran2@udi.edu.co

Manuscrito recibido el 21 de septiembre de 2020. Aceptado, tras revisión, el 15 de marzo de 2021. Publicado el 1 de septiembre de 2021.

\begin{abstract}
Resumen
La complejidad de los problemas actuales del campo colombiano pone en riesgo la sostenibilidad de las Unidades de Producción Agropecuaria (UPA) familiares. En consecuencia, se convierte en un área de estudio fundamental para el mejoramiento de la economía rural. Debido a ello, se desarrolló una investigación documental y de campo sobre un caso de estudio ubicado en Lebrija, Colombia, para el análisis sistémico de la sostenibilidad económica en 10 UPAS promotoras de la agricultura sostenible. Los resultados permitieron establecer la efectividad del ES en la caracterización de UPAs. Se identificó que la falta de inversión en infraestructura y tecnologías apropiadas han hecho que las UPAs destinen más del $50 \%$ de su área total para el inadecuado pastoreo de bovinos. Esta distribución de la tierra amenaza la sostenibilidad de la economía de las familias locales, dado que la producción y rentabilidad de la ganadería no son suficientes para su sustento y el mantenimiento de los potreros. El inadecuado pastoreo genera grandes cantidades de estiércol que contamina el medio ambiente. Lo anterior afecta la congruencia entre las actividades productivas y los principios filosóficos de las UPAs.
\end{abstract}

Palabras clave: Desarrollo rural, enfoque sistémico, excedentes orgánicos, sostenibilidad económica, compost.

\begin{abstract}
The complexity of the current problems in the Colombian countryside puts at risk the sustainability of family Agricultural Production Units (UPA for its acronym in Spanish). Consequently, it becomes a fundamental study area for the improvement of the rural economy. For this reason, a documentary, field investigation was developed on a case study located in Lebrija, Colombia, for the systemic analysis of economic sustainability in 10 UPAS promoters of
\end{abstract}


sustainable agriculture. The results allowed to establish the effectiveness of the ES in the Characterization of UPAs. It was identified that the lack of investment in infrastructure and appropriate technologies has made the UPAs to allocate $50 \%$ of its total area for inadequate grazing. This distribution of land threatens the sustainability of the economy of local families, given that the production and profitability of livestock are not enough for their livelihood and the maintenance of pastures. The inadequate grazing generates large amounts of manure that pollutes the environment. The above affects the congruence between the productive activities and the philosophical principles of the UPAs.

Keywords: Rual development, systemic approach, organic surpluses, economic sustainability, compost.

Forma sugerida de citar: Castro-Castro, M., Beltrán-Díaz, A. y Vargas-Espítia, A. (2021). Análisis sistémico de la sostenibilidad económica de unidades de producción agropecuaria familiar en una comunidad campesina de Lebrija, Colombia. La Granja: Revista de Ciencias de la Vida. Vol. 34(2):141-153. http://doi.org/10.17163/lgr.n34.2021.10.

IDs Orcid:

Martha Lenis Castro-Castro: http:/ / orcid.org/000-0001-7897-1040

Albeiro Beltrán-Díaz: http:/ / orcid.org/0000-0002-1585-0708

Adolfo Vargas-Espítia: http:/ / orcid.org/0000-0003-4741-4991 


\section{Introducción}

Las fincas de la vereda la cuchilla del municipio de Lebrija Santander representan un conjunto de Unidades de Producción Agropecuaria (UPAs) familiares, vinculadas a una asociación de mujeres campesinas llamada AMMUCALE (Díaz, Ortiz y Rodríguez, 2011). Sus propietarias promueven principios de protección del agua, los bosques, los saberes ancestrales, las semillas nativas y la soberanía alimentaria, en estrecha relación con los principios del biocentrismo, las economías alternativas (trueque) y la vida sencilla que expresan los Objetivos del Buen Vivir - OBV (Cubillo e Hidalgo, 2019; Huanacuni, 2010). Si bien son un ejemplo local de agricultura responsable alineada con algunos Objetivos de Desarrollo Sostenible - ODS (ONU, 2015) según diferentes estudios (Díaz, Ortiz y Rodríguez, 2011; Amaya, Mendez y Hernández, 2018; Cruz y col., 2018), sufren parte de los problemas del sector rural colombiano, circunstancia que las ha convertido como caso de estudio.

La distribución y posesión de la tierra demuestra que el $70 \%$ de las UPAs colombianas poseen menos de 5 ha (DANE, 2015). Esta atomización limita la capacidad de producción de las fincas (Kalmanovitz y López, 2003). Del mismo modo, temas como la inversión en maquinaria, infraestructura, sistemas de riego y asistencia técnica se mantienen por debajo del 15\% (DANE, 2015), mientras que el uso de fertilizantes artificiales es 2,83 veces mayor al promedio suramericano (Banco Mundial, 2019a). Esto dificulta a los campesinos a mantener estables sus costos de producción y pone en riesgo su sostenibilidad económica.

Si bien, desde los años 50 se han intentado mejorar las condiciones del agro mediante los planes de desarrollo (Kalmanovitz y López, 2003); los resultados han demostrado un aumento en la brecha de desigualdad entre el campo y la ciudad con un Gini (Banco Mundial, 2019b) del 0,45 (DANE, 2019). Algunas investigaciones (Arias, Mader y Escobar, 2008; Ruiz y Oregui, 2001), exponen que una de las razones principales es que tradicionalmente se ha observado el agro desde un enfoque reduccionista, lo que resulta en una visión parcial de los problemas. En respuesta, algunos autores (Bistagnino, 2011; Capra, 1996; Meadows, 2008; Rovaletti, 1989) señalan la necesidad de cambiar a un enfoque sisté- mico - ES para el análisis de situaciones complejas, en donde diferentes investigaciones aplicadas han validado el ES para el diseño de modelos de toma de decisiones (Stamberg, 2015) y de producción sostenible (Barbero y Toso, 2006).

De acuerdo a lo anterior, el objetivo principal del estudio es realizar un análisis sistémico de la sostenibilidad económica en 10 UPAs promotoras de la agricultura sostenible. Para ello se proponen los objetivos específicos: (1) realizar un análisis sistémico de las UPAs en relación con el territorio basado en la metodología Sistemic Desing de Bistagnino (2009), (2) efectuar una revisión detallada de los resultados económicos en materia de producción y rentabilidad y (3) identificar las problemáticas sistémicas y sus posibles oportunidades de mejora.

\section{Materiales y Métodos}

El levantamiento de información a través de fuentes primarias y secundarias, el análisis sistémico e interpretación de los datos se realizó entre mayo y diciembre del año 2019 a partir de un estudio de caso, aplicando un análisis cualitativo y cuantitativo.

\subsection{Caso de estudio}

El área de estudio se encuentra en zona rural del municipio de Lebrija - Santander, nororiente de Colombia. En esta región el $80 \%$ de sus habitantes deriva sus ingresos de actividades agropecuarias, principalmente en cultivos de piña, limón Tahití y cacao. El 84,27\% de las UPA poseen menos de 20 ha (Alcaldía de Lebrija, 2016); tal como se observa en la Figura 1. Las fincas del territorio poseen en promedio 20 ha, cuyas actividades productivas giran en torno al cultivo de cítricos, vegetales, cacao y al pastoreo de ganado.

Para la selección del caso de estudio se usaron criterios tales como: la extensión del predio, tipos de cultivos, actividades pecuarias, ubicación y orientación hacia la sostenibilidad. Se seleccionaron 10 UPAs ubicadas en la vereda la cuchilla, encabezadas por la finca "Tierra Buena". Sus administradoras son mujeres quienes son cabeza de familia pertenecientes a AMMUCALE, las cuales cumplen con las características necesarias para servir como una muestra representativa de la asociación. 

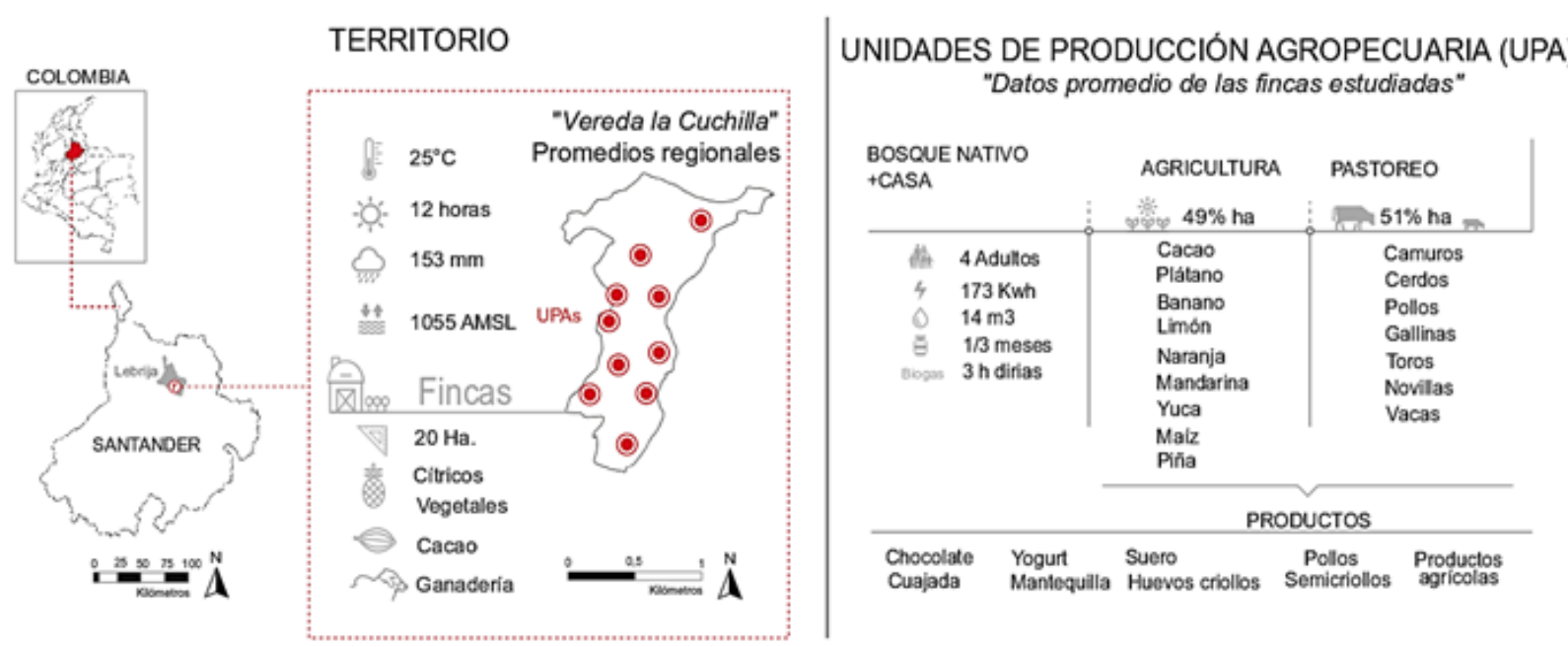

Figura 1. Promedios del territorio y UPAs caso de estudio basado en los datos de la Alcaldía de Lebrija (2016).

En esta zona, las UPAs seleccionadas desarrollan procesos productivos que agregan valor sus productos. El 100\% del cacao es transformado en chocolate y las heces porcinas son aprovechadas para la generación de gas a partir de biodigestores. Sus ingresos se encuentran diversificados mediante 5 actividades pecuarias y 8 agrícolas, comercializadas bajo prácticas de comercio justo (Díaz, Ortiz y Rodríguez, 2011; Amaya, Mendez y Hernández, 2018; Cruz y col., 2018). Adicionalmente, la finca Tierra Buena lidera los procesos agroecológicos de la vereda, dada su antigüedad dentro de AMMUCALE y la visión humana de su propietaria.

\subsection{Método}

\subsubsection{Análisis sistémico}

Se aplicó el ES según la metodología sugerida por Bistagnino (2011), la cual consiste en el análisis del territorio, los actores y los sistemas a nivel macro, meso y micro (Ceschin, 2014), buscando identificar y caracterizar en términos cuantitativos y cualitativos las entradas, procesos, salidas, las problemáticas económicas (Barbero y Toso, 2006; Bertalanffy, 1968; Bistagnino, 2011; Capra, 1996; Carrá, 1961; Garciandía, 2011; Johansen, 1993; Meadows, 2008; Stamberg, 2015; Rovaletti, 1989) y los puntos de apalancamiento (Meadows, 1997) para el mejoramiento de la sostenibilidad según James (2015).

En la primera etapa se realizó el análisis del te- rritorio mediante la revisión de fuentes secundarias (artículos, libros, literatura gris, sitios web oficiales) para obtener datos sobre las características culturales, geográficas y productivas del agro lebrijense.

En la segunda etapa, el análisis de los actores se realizó usando como instrumento de recolección de información una entrevista semiestructurada, la cual fue aplicada a 42 personas involucradas directamente en las actividades agropecuarias del caso de estudio, como sus propietarias, sus hijos y sus cónyuges. Las categorías de análisis corresponden a la dimensión económica de la sostenibilidad basado en James (2015), la producción agropecuaria, la economía familiar y los procesos de transformación de las materias primas.

En la tercera y última etapa, para la caracterización de los subsistemas se usó como instrumento la observación y como herramienta para la estructuración y comprensión de los datos el mapa de sistemas (Vargas y col., 2020; Vezzoli y col., 2014). Finalmente, debido a la heterogeneidad de la información recopilada con el ES se entrevistaron a tres expertos en producción responsable, etnobiología y comercio justo, quienes aportaron su interpretación sobre los resultados del caso de estudio relacionados con agroecología, tejido social y comercio agrícola informal. 


\subsubsection{Análisis de dimensión económica de la sosteni- bilidad.}

Para el análisis de la dimensión económica se adaptó el Profile Questionnaire diseñado por (James, 2015), el cual consta de 49 preguntas, distribuidas equitativamente sobre siete temas relacionados con: 1) producción y dotación de recursos, 2) intercambio y transferencia, 3) contabilidad y regulación, 4) consumo y uso, 5) trabajo y bienestar, 6) tecnología e infraestructura, 7) riqueza y distribución. La entrevista fue aplicada a las propietarias de las fincas, sus cónyuges y sus hijos mayores de edad, para un total de 42 personas. Las opciones de respuesta buscaron comprender la percepción de los entrevistados sobre cada uno de los siete temas expuestos, quienes en cada pregunta escogieron una única respuesta entre crítico, malo, muy insatisfecho, insatisfecho, básico, satisfactorio, muy satisfactorio, bueno y excelente, las cuales fueron tabuladas en una tabla de Excel y asignadas a un valor de 1 a 9, donde 1 corresponde a crítico y 9 a excelente. Se promediaron las respuestas con el fin de obtener la percepción por cada una de las 49 preguntas. Posteriormente, se calculó el promedio de satisfacción para cada uno de los siete temas abordados. Finalmente, se calculó la percepción general de los encuestados sobre la sostenibilidad económica de sus familias. Los resultados fueron plasmados gráficamente para observar los niveles de los siete temas.

\subsubsection{Análisis de rentabilidad del eje económico.}

Se aplicó un enfoque cuantitativo centrado en el valor económico de los bienes producidos en las UPAs. Para ello, se recolectó información sobre la producción de los microsistemas en función del dinero, y se registró en tablas de cálculo. La capacidad económica se evaluó mediante el valor agregado neto (VAN) (Stamberg, 2015), el cual se halló mediante la fórmula (1).

$$
\mathrm{VAN}=\mathrm{PB}-\mathrm{CI}-\mathrm{D}
$$

Donde PB es la producción bruta de las UPAs en pesos colombianos; CI es el consumo intermedio o el costo de los insumos adquiridos; D es la suma de la depreciación de las máquinas, equipos e instalaciones utilizadas en la producción de bienes y servicios.

Adicionalmente, se halló la rentabilidad agropecuaria RA que permite conocer el rendimiento del negocio después de pagar salarios (S), intereses bancarios (J), arrendamientos (T) e impuestos (I) (Stamberg, 2015), por lo que permite obtener una visión realista sobre los beneficios finales percibidos por las familias. Para el cálculo de la RA, se aplicó la fórmula de Stamberg (2015) (2).

$$
\mathrm{RA}=\mathrm{VAN}-\mathrm{S}-\mathrm{J}-\mathrm{T}-\mathrm{I}
$$

Las formulas fueron aplicadas de acuerdo a las dos actividades desarrollada en las UPAs. La primera abarca todas las actividades de tipo agrícola; la segunda incluye únicamente la actividad ganadera. Los datos fueron tabulados y analizados en Excel, en donde se desarrolló un análisis descriptivo de las variables, se aplicaron las formulas y se obtuvieron totales promedio por tipo de actividad.

\section{Resultados}

\subsection{Problemas identificados mediante el ES}

Mediante el uso del ES en las observaciones de campo se detalló a nivel micro la forma en que fluyen los recursos a través de los sistemas productivos de las UPAs; esto permitió identificar cinco problemas comunes en las fincas estudiadas, tres de ellos relacionados con las entradas, uno en los procesos y uno más en las salidas, situaciones que se configuran en una amenaza para la consecución de una zona rural sostenible (Figura 2).

En las entradas se hallaron tres situaciones $\mathrm{cu}-$ yos impactos resultan ser negativos para la sostenibilidad de las fincas estudiadas. La primera de ellas se refiere a la falta fertilizantes. Si bien las propietarias promueven el rechazo a los agroquímicos, no se evidenció el uso de abonos orgánicos, lo que en el largo plazo podría generar dificultades en la producción agrícola por la disminución de nutrientes en el suelo. La segunda se relaciona con la baja rentabilidad de las fincas, dado que el ingreso económico no es suficiente para cubrir los costos y las necesidades familiares. La tercera se refiere a los ingresos ajenos a la producción agropecuaria. En 8 de las 10 UPAs estudiadas, la mayor parte del ingreso económico proviene de empleos urbanos de los cónyuges e hijos. Esto último es beneficioso para la economía del hogar, pero a largo plazo podría desplazar a estas familias hacia las zonas urbanas en busca de mejores oportunidades. 


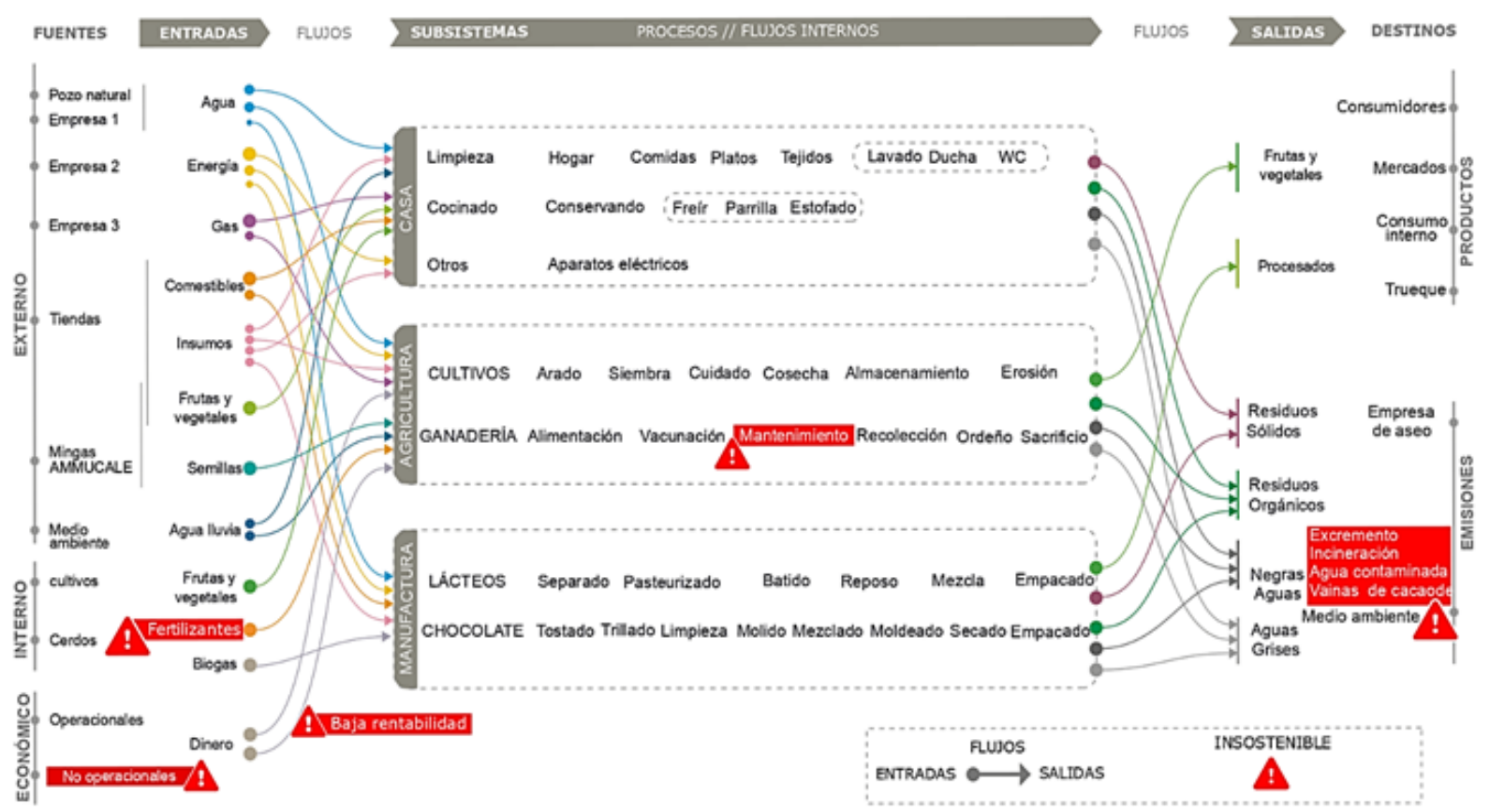

Figura 2. Análisis a partir del Mapa del sistema actual de la UPA. Los símbolos de alerta reflejan problemas identificados en relación a la sostenibilidad.

En los procesos se identificaron problemáticas relacionadas con el mantenimiento de las UPAs, específicamente en las zonas de potrero. Se evidenció que en 7 de las 10 UPAs estudiadas, el uso del suelo exclusivo para el pastoreo de bovinos es mayor al 50\%; sin embargo, la producción lechera y la venta de ejemplares no generan los ingresos suficientes para cubrir los costos de mantenimiento asociados con la restauración de postes, cercas, el pago de impuestos, vacunas, alimentación, entre otros. Aunque la vereda la cuchilla es una zona que tradicionalmente combina actividades agrícolas con ganadería, se evidenció que en las fincas estudiadas la cantidad de ejemplares corresponde en promedio a 1 res por cada 1,5 ha.

En las salidas se evidenció una problemática relacionada con los excedentes orgánicos y el medio ambiente. A partir de las observaciones de campo se logró identificar que el estiércol del ganado, las vainas del cacao, las aguas residuales del hogar y algunas basuras reciben una inadecuada disposición, y como resultado terminan convirtiéndose en un foco de contaminación ambiental. En el caso del estiércol del ganado, se logró identificar que en las fincas estudiadas se generan aproximadamente 219,55 t de estiércol septimestralmente, las cuales son dispersas por los animales sobre los potreros y no reciben ningún tipo de manejo. Del mismo modo, durante los meses de medición de excedentes, se identificó que en las fincas estudiadas, la producción de cacao generó 53,9 t de vainas, las cuales se acumularon en montones dentro del cultivo y, al igual que el estiércol, no recibieron ningún manejo adecuado.

De igual manera, se identificó que las aguas residuales de los hogares son vertidas al medio ambiente sin ningún tipo de tratamiento de descontaminación, cuya cifra se desconoce debido a la falta de acueducto, contadores y alcantarillado en la zona, pero a partir del promedio colombiano de consumo por familia (EPM, 2020) se estima podría aproximarse a los 115,5 metros cúbicos mensuales por hogar. Finalmente, se evidenció que los excedentes inorgánicos como bolsas plásticas, envolturas de alimentos, papel higiénico entre otros, son incinerados por algunas familias, debido a que el servicio de recolección de basuras no presta sus servicios en zonas rurales; por lo que no fue posible estimar el 
nivel de contaminación que esta práctica genera, sin embargo, se consideró como un foco de contaminación relevante para mencionar en los resultados del ES.

\subsection{Análisis de la Sostenibilidad económica de las UPAs}

Gracias a la aplicación de la adaptación del Profile Questionnaire de James (2015), se identificó que la percepción de las familias hacia los temas abordados deja al eje económico de la sostenibilidad en un nivel general básico. Sin embargo, al observar ca- da una de sus aristas de forma particular, es posible observar que algunos de los temas que componen a esta dimensión se encuentran en niveles más bajos. Como se aprecia en la Figura 3, una de las principales falencias que poseen las UPAs estudiadas y que se encuentra en un nivel malo es su falta de estrategias contables y regulación, tema que, según los entrevistados, dificulta la posibilidad de conocer la forma en que fluye el dinero dentro de la economía familiar y la transparencia en el cómo se usa. Así mismo, este desconocimiento contable impide que las familias puedan diseñar planes a mediano y largo plazo, limitándolos a una explotación económica cortoplacista.

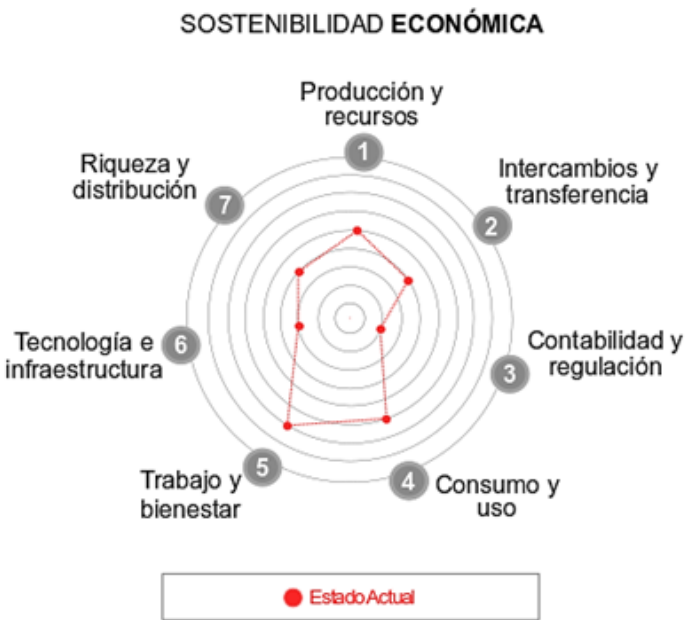

Figura 3. Perfil de la sostenibilidad económica de las UPAs, adaptado de (James, 2015).

Por su parte, la arista de tecnología e infraestructura posee un nivel muy insatisfactorio, principalmente por la falta de dotación y disposición de estas herramientas y una debida adecuación de la infraestructura actual enfocada hacia el desarrollo sostenible; es decir, los campesinos encuestados consideran que no les ha sido posible acceder a nueva tecnología ni mejorar la que poseen actualmente.

Así mismo, según los resultados de la entrevista, las aristas 7 y 2 relacionadas con la riqueza y los intercambios se ubicaron en el nivel insatisfactorio. Esto implica una informidad por parte de los habitantes de la zona sobre las oportunidades de comercio a su disposición y la retribución de la riqueza.
Finalmente, las aristas 1,4 y 5 relacionadas con la producción, el consumo y el empleo se posicionaron entre los niveles básico y muy satisfactorio. Esto significa que los entrevistados tienen una percepción entre normal y positiva sobre estos tres temas, principalmente por la labor que sus propietarias han desarrollado con AMMUCALE desde hace más de 20 años y que ha promovido la diversificación de la producción, el consumo responsable y el compromiso de los jóvenes con el campo.

\subsection{Rentabilidad de las UPAs}

Como parte de la comprensión de la dimensión económica de la sostenibilidad, se realizó el cálculo del 
valor agregado neto y la rentabilidad agropecuaria de cada una de las UPAs estudiadas. Como se puede observar en la Figura 4, los resultados permitie- ron identificar las actividades más y menos productivas, así como el beneficio generado de acuerdo al espacio requerido por cada una de ellas.

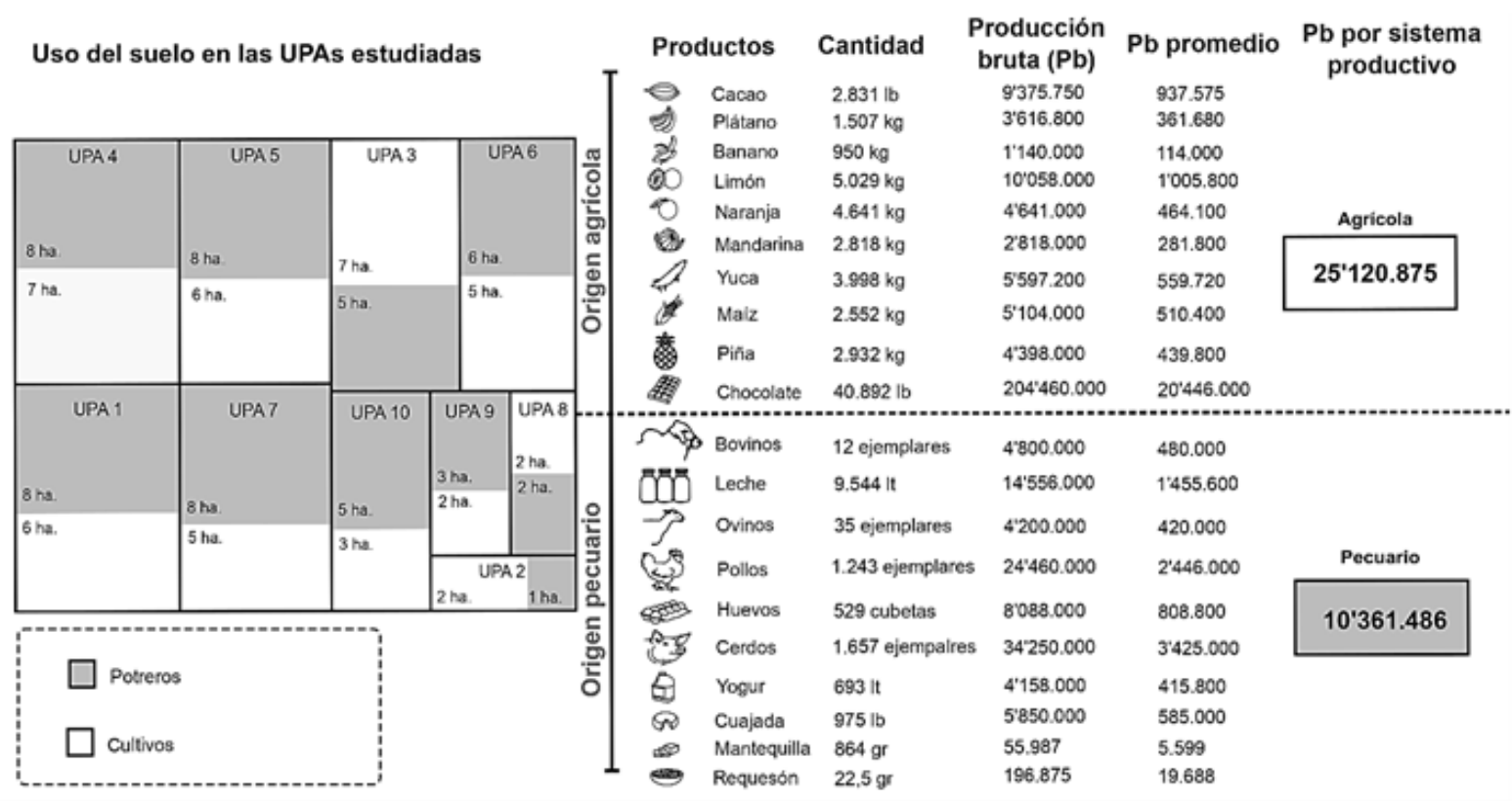

Figura 4. Uso de suelo y producción bruta $(\mathrm{Pb})$ de las UPAs estudiadas

Aunque el municipio de Lebrija no es una zona ganadera, en las UPAs estudiadas se usa cerca del $56 \%$ del suelo como potrero, en el cual se mantienen pocos ejemplares por hectárea. La venta de ganado no es la actividad económica más representativa, ya que los bovinos son usados principalmente para la producción de leche y derivados como yogur, cuajada, mantequilla y requesón; el ganado es visto como una fuente de ahorro de capital. En la figura 4 se puede apreciar que la actividad pecuaria que más genera ingresos es la cría de cerdos, seguida por la venta de pollo criollo y la comercialización de leche; la ganadería por su parte genera un ingreso promedio de $\$ 480.000$ por UPA al año, ocupando el sexto puesto en las actividades pecuarias y el onceavo a nivel general.

En esta zona se han impulsado proyectos de manufactura que buscan agregar valor a las materias primas, como en el caso del chocolate y los derivados de la leche. En este aspecto, la elaboración de chocolate de mesa es la actividad que más aporta a la economía familiar, razón por la cual la mayor parte del cacao es transformado y solo cerca de $2.831 \mathrm{lb}$ son vendidas en grano.

A pesar que la producción por actividad y el uso de suelo permiten comprender algunos aspectos de la economía local, no reflejan por completo la realidad de los hogares. En la Tabla 1 se muestra de manera consolidada los resultados en materia de rentabilidad (RA), donde después de restar los costos asociados a cada sistema productivo, se identificó cuánto dinero realmente reciben en promedio las UPAs y cuánto es el aporte por hectárea de acuerdo a su uso.

Los resultados que muestra la Tabla 1 corresponden a los valores promedio de las 10 UPAs. Como se puede apreciar, las actividades derivadas de la producción agrícola presentan un VAN promedio de $\$ 19^{\prime} 988.115$ y una RA de $\$ 13^{\prime} 146.944$, los cuales se traducen en una rentabilidad por hectárea de $\$ 4^{\prime} 537.427$. Sin embargo, las actividades pecuarias, 
dentro de las cuales se encuentra la ganadería y sus derivados, presentan en promedio una rentabilidad por hectárea de $\$ 497.365$.

En 8 de las 10 Upas estudiadas, el área usada como potreros fue mayor que el área usada para cul- tivos agrícolas. A pesar de disponer de un mayor espacio, el beneficio económico recibido por la unidad familiar como resultado de las actividades pecuarias llega a $\$ 3^{\prime} 098.579$ de pesos al año, mucho menor que la generada por las actividades agrícolas.

Tabla 1. VA y RA promedio de las UPAs estudiadas

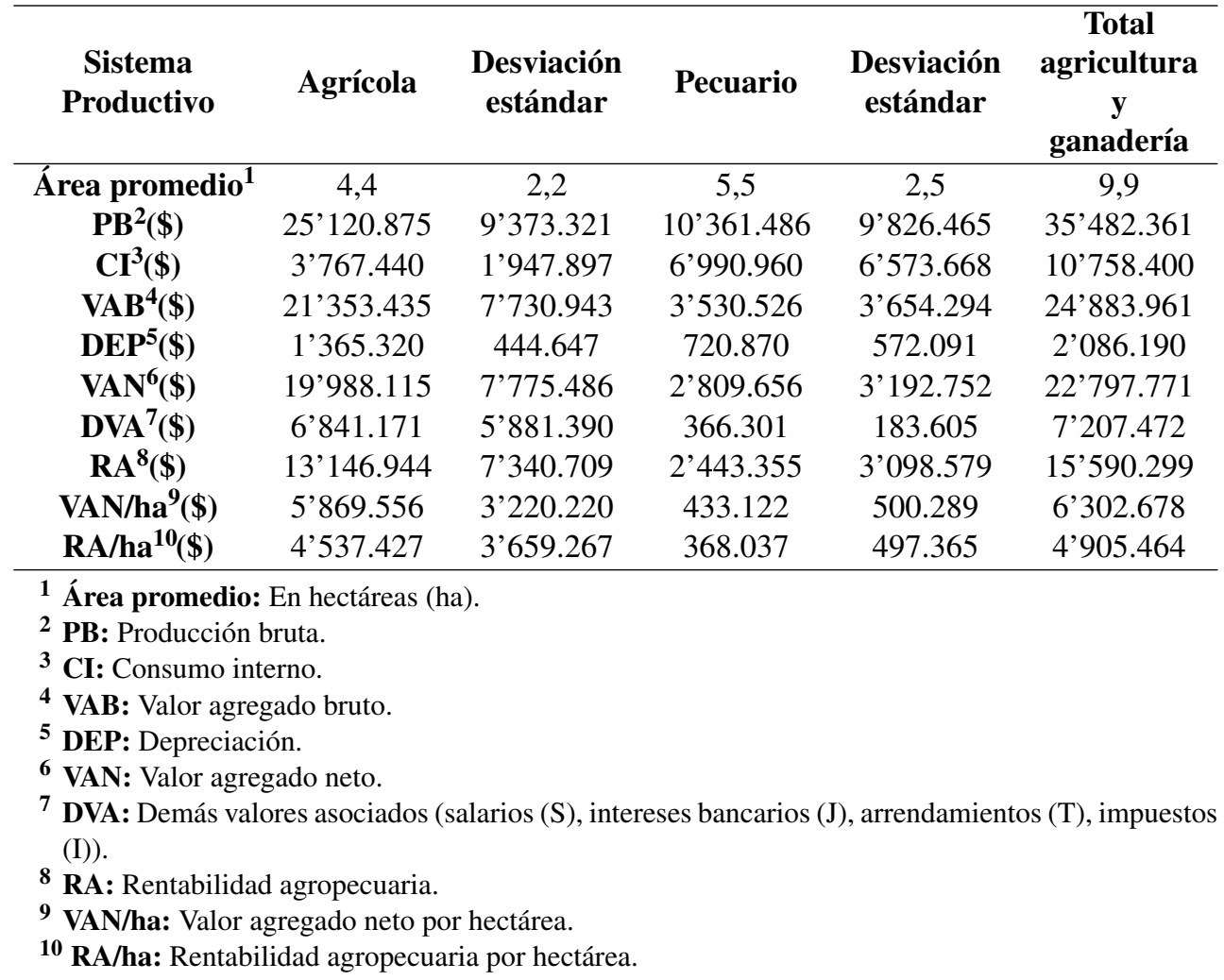

Esto se debe principalmente al espacio destinado a la ganadería y los costos relacionados con alimentos y vacunas que requieren los demás animales para su desarrollo. Por su parte, se pudo evidenciar que las actividades agrícolas, incluyendo la trasformación del cacao en chocolate, generan en promedio \$13'146.944 al año, siendo la principal fuente de ingresos y generación de valor de las familias campesinas de la zona.

\section{Discusión}

\subsection{El ES para el análisis de UPAs}

El ES permitió comprender de forma holística la situación económica y la identificación de problemas asociados con la incorrecta disposición del estiércol del ganado y las vainas de cacao en las UPAs familiares. Esto se apoya en otras investigaciones como la de Steinfeld y col. (2009) quienes aseguran que estos materiales contaminan al medio ambiente. Además, Sosa y García (2019) afirman que el estiércol bovino produce gases de efecto invernadero y Pinos y col. (2012) y Steinfeld y col. (2009) aseguran que este excedente genera una serie de micro y ma- 
cro nutrientes que afectan negativamente al suelo y los acuíferos.

Del mismo modo, el ES permitió identificar contracciones filosóficas entre las actividades que desarrollan las UPAs estudiadas y los ideales que promulgan sobre agricultura sostenible y protección del medio ambiente. Esto es similar a los resultados de un caso publicado por Jagustović y col. (2019), en el cual una comunidad de mujeres agricultoras en Doggoh-Jirapa, norte de Ghana, a través de ES identificaron elementos transdisciplinarios contradictorios a sus principios de Climate-Smart Agriculture -CSA y les permitió diseñar estrategias de mejora.

El ES también permitió identificar los excedentes orgánicos como el estiércol del ganado, las vainas de cacao y otros elementos vegetales que pueden ser aprovechados por los campesinos de la región en la elaboración de vermicompost, el cual es rico en microorganismos (Asadu y col., 2019; Barbero y Toso, 2006; FAO, 2013). Esto es beneficioso para los cultivos, debido a que contiene grandes cantidades de bacterias fijadoras de nitrógeno que mejora la retención de agua, la salud biológica y la capacidad de absorción del suelo, el aprovechamiento de las aguas pluviales, la actividad enzimática, la presencia de nutrientes, entre otros (Agegnehu y col., 2016; Argaw, 2017; Rayen y col., 2006; Sharma y col., 2017; Wang y col., 2016); lo que podría convertirse en una oportunidad para solucionar los problemas identificados en materia de contaminación y para apoyar el desarrollo de la agricultura responsable y sostenible.

\subsection{Sobre la dimensión económica de la sostenibilidad en comunidades rurales}

En este punto, el estudio pretende abrir una discusión basada en el uso del suelo y los valores del VAN y la RA, los cuales sugieren la potencialización de la actividad agrícola por encima de algunas actividades pecuarias como la ganadería. Esto se asemeja a la propuesta de Stamberg (2015), quien en su investigación mediante el ES aconsejó la potencialización de una actividad y la eliminación de otra a partir de los resultados de VAN y RA. Como se pudo evidenciar, la RA del sistema pecuario, en la forma cómo se desarrolla actualmente la ganadería en la UPAs estudiadas y en comparación con las actividades agrícolas, no genera ingresos suficientes para beneficiar a las familias, lo que abre la puerta a futuras investigaciones sobre formas sostenibles de aprovechar el suelo en la ruralidad, considerando temas como la diversificación de cultivos, la cantidad de reses por hectárea y el uso de establos (Rojas, Botero y Osorio, 2013). Esto último se apoya en la propuesta de Fernández y col. (2016) sobre los establos adecuados que facilitan la disposición de las excretas.

Lo anterior sugiere además la necesidad de crear un sistema completo de gestión de excedentes orgánicos que permita el aprovechamiento del estiércol bovino, las vainas de cacao y otros excedentes vegetales, mediante procesos de compostaje. Esto resalta la necesidad de adelantar estudios enfocados en el diseño de infraestructura adecuada y el asesoramiento en el manejo de estos materiales.

Se espera que esta investigación sea un insumo para la toma de decisiones de las propietarias de las UPAs estudiadas, en el mejoramiento de su ingreso neto, el impulso de su independencia económica, su empoderamiento, el fortalecimiento de su identidad, su compromiso como mujer campesina (Botello y Guerrero, 2017) y además sirva como base para futuras investigaciones relacionadas con economía y desarrollo rural sostenible.

\section{Conclusiones}

La investigación aporta a la literatura información sobre la aplicación del ES al análisis de UPAs, principalmente por la atención particular que este enfoque presta a los flujos y su interacción con los actores involucrados, lo que conllevó a la identificación de la falta de asesoramiento en prácticas sostenibles y por lo cual, algunas de ellas contradicen sus ideologías. Así mismo, se reconocen algunas limitantes propias de este enfoque, dado que exige la recolección de grandes cantidades de información, y por consiguiente demanda mayores cantidades de tiempo, recursos y asesoramiento de expertos en múltiples disciplinas.

La falta de organización y registros contables impide que los propietarios de las UPAs sean conscientes de la forma en que fluye el dinero a través de sus negocios y se considera como una limitante 
para el diseño de planes y estrategias de mejora, que en su mayoría requieren de recursos económicos con los que no cuentan las familias campesinas; además, la falta de inversión pública y el difícil acceso al crédito reduce aún más las posibilidades de los campesinos en la obtención de tecnología, asesoría e infraestructura adecuada.

Finalmente, se concluye que el uso del suelo en Colombia es un problema que se refleja en la distribución productiva aplicada por las UPAs familiares, donde la mayor parte del área es utilizada para el pastoreo de bovinos que, en promedio, gozan de 1,5 ha por ejemplar. Para el desarrollo de la agricultura sostenible es necesario promover métodos eficientes que permitan mejorar la economía familiar y disminuir las brechas de desigualdad entre el campo y la ciudad, además de garantizar el incremento del suelo agrícola, como estrategia de seguridad alimentaria para el sostenimiento de las futuras generaciones.

\section{Agradecimientos}

Se agradece a Minciencias y a la Corporación Universidad de Investigación y Desarrollo- UDI, financiadores de la investigación mediante el convenio 382-2019. Además, se reconoce la colaboración prestada por AMMUCALE en cabeza de Psic. Tailana Prieto; los actores relacionados con el caso de estudio, la señora Rosa Isabel Rincón, Román Bastos y la señora Zoraida Uribe. Adicionalmente, se agradece a los expertos consultados: Msc. Melissa Ayala asesora en etnobiología, Ing. Luz Holanda asesora en producción responsable y Econ. Adolfo Botero asesor en comercio justo.

\section{Referencias}

Agegnehu, G. y col. (2016). «Benefits of biochar, compost and biochar-compost for soil quality, maize yield and greenhouse gas emissions in a tropical agricultural soil». En: Science of The Total Environment 543, 295-306. Online: https: / / bit. ly/3uEIZyI.

Alcaldía de Lebrija (2016). «Economía». Online: https:/ / bit.ly/3vV8LPm.
Amaya, M., R. Mendez y A. Hernández (2018). «Revisión documental sobre la experiencia de la Asociacion Municipal de Mujeres Campesinas de Lebrija Santander "AMMUCALE"». Tesis de Grado. Universidad Industrial de Santander. Online: https:/ / bit.ly/3tSr1Y3.

Argaw, A. (2017). «Organic and inorganic fertilizer application enhances the effect of Bradyrhizobium on nodulation and yield of peanut (Arachis hypogea L.) in nutrient depleted and sandy soils of Ethiopia». En: International Journal of Recycling of Organic Waste in Agriculture 6.3, 219-231. Online: https://bit.ly/3f8GXzZ.

Arias, R., T. Mader y P. Escobar (2008). «Factores climáticos que afectan el desempeño productivo del ganado bovino de carne y leche». En: Archivos de medicina veterinaria 40.1, 7-22. Online: https:/ / bit.ly/3tYFPVl.

Asadu, C. y col. (2019). "Survey on solid wastes management by composting: Optimization of key process parameters for biofertilizer synthesis from agro wastes using response surface methodology (RSM)». En: Artificial Intelligence in Agriculture 3, 52-61. Online: https : / / bit.ly / 2RzX8hQ.

Banco Mundial (2019a). «Índice de Gini - Colombia». Online: https:/ / bit.ly/3o0ndTe.

- (2019b). «Uso de fertilizantes por país». Online: https:/ / bit.ly/2R7yNzV.

Barbero, S. y D. Toso (2006). «Systemic Design of a Productive Chain: reusing coffee waste as an input to agricultural production». En: Environmental Quality Management, 24-46. Online: https: / / bit.ly/3tSj70Y.

Bertalanffy, L. Von. (1968). General System Theory: Foundations, Development, Applications. George Braziller.

Bistagnino, L. (2009). «Progettare la sostenibilità produttiva e ambientale in agricoltura, industria e comunità locali». En: Slow Food Editore. Cap. Systemic Design sistemico.

- (2011). Systemic design: designing the productive and environmental sustainability. Politecnico di Torino. Online: https:/ / bit.ly/3y8Wt7R.

Botello, H. e I. Guerrero (2017). «Condiciones para el empoderamiento de la mujer rural en Colombia». En: Entramado 13.1, 62-70. Online: https:/ / bit.ly/3eG1PzH.

Capra, F. (1996). The Web of Life: A New Scientific Understanding of Living Systems. Anchor Books. 
Carrá, J. C. (1961). «Pensamiento Sistémico». Online: https://bit.ly/2RSJ571.

Ceschin, F. (2014). Sustainable product-service systems: Between strategic design and transition studies. Springer Science y Business Media.

Cruz, E. y col. (2018). "Mujeres por el buen vivir, perspectivas desde la investigación para la paz. Asociación municipal de mujeres campesinas del municipio de Lebrija "AMMUCALE"». Tesis de Grado. Universidad Industrial de Santander. Online: https:/ / bit.ly/2QooF5J.

Cubillo, A. y A. Hidalgo (2019). «Una propuesta para la construcción de un transdesarrollo global . Los Objetivos del Buen Vivir». Online: https: / / bit.ly/3ydVi6X.

DANE (2015). Censo Nacional Agropecuario (CNA). Resultados Nacionales Con Resúmenes Provinciales CNA 2000. Online: https://bit.ly/3w4DNnW.

- (2019). Boletín Técnico Pobreza monetaria en Colombia año 2018. Inf. téc. Departamento Administrativo Nacional de Estadística. Online: https:/ / bit. ly/3y99Qoy.

Díaz, L., J. Ortiz y D. Rodríguez (2011). «Propuesta para el fortalecimiento de las condiciones socioeconómicas de la zona de influencia de ammucale" (Sector rural del municipio de Lebrija)». Tesis de Grado. Universidad Industrial de Santande. Online: https:/ / bit.ly/33G9M1z.

EPM (2020). «Tips para el uso inteligente». Online: https://bit.ly/3y9cyKK.

FAO (2013). Manual de compostaje del agricultor. Oficina Regional de la FAO para América Latina y el Caribe. Online: https:/ /bit.ly/33IOb8y.

Fernández, C. y col. (2016). Establo sostenible. Evaluación en granjas de vacuno de leche como estrategia para la mejora de la rentabilidad. Inf. téc. 122. Afriga. Online: https://bit.ly/3foKWIX.

Garciandía, J. (2011). Pensar sistémico, una introducción al pensamiento sistémico. Pontificia Universidad Javeriana. Online: https://bit.ly/3flcnn8.

Huanacuni, F. (2010). Buen Vivir / Vivir Bien Filosoía, políticas, estrategias y experiencias regionales andinas. Coordinadora Andina de Organizaciones Indígenas (CAOI). Online: https: / / bit.ly / 3hx7muo.

Jagustović, R. y col. (2019). «Contribution of systems thinking and complex adaptive system attributes to sustainable food production: Example from a climate-smart village». En: Agricultural systems 171, 65-75. Online: https: / / bit.ly / 2SMmN7r.
James, P. (2015). Urban sustainability in theory and practice: circles of sustainability. Routledge. Online: https://bit.ly/3fDJcMj.

Johansen, O. (1993). Introducción a la teoría general de sistemas. Editorial Limusa. Online: https: / / bit. ly/2RWe2Yj.

Kalmanovitz, Sa. y E. López (2003). «La agricultura en Colombia entre 1950 y 2000». En: Revista del Banco de la República 76.912, 1-45. Online: https: //bit.ly/3fs2808.

Meadows, D. (1997). Lugares donde intervenir en un sistema. Whole Earth.

- (2008). Thinking in systems: A primer. Chelsea green publishing.

ONU (2015). «Objetivos de desarrollo sostenible». Online: https:/ / bit.ly/2SNQOnn.

Pinos, J. y col. (2012). «Impactos y regulaciones ambientales del estiércol generado por los sistemas ganaderos de algunos países de América». En: Agrociencia 46.4, 359-370. Online: https: / / bit.ly/ $3 \mathrm{w} 7 \mathrm{vN5B}$.

Rayen, M. y col. (2006). «Efecto de la adición de compost sobre propágulos micorrícicos arbusculares en un suelo volcánico del centro sur de Chile». En: Revista de la ciencia del suelo y nutrición vegetal 6.3, 26-39. Online: https: / / bit.ly / 3ycITQQ.

Rojas, D., J. Botero y O. Osorio (2013). «Análisis técnico económico de sistemas de ganadería en confinamiento modelo establo invernadero en el departamento del Quindío». En: Sinapsis 5.5, 127-136. Online: https://bit.ly/2QnUMm0.

Rovaletti, M. L. (1989). «Teoría general de los sistemas». En: Signo y Pensamiento 8.15, 45 -56. Online: https://bit.ly/3w9mXnR.

Ruiz, R. y L. Oregui (2001). «El enfoque sistémico en el análisis de la producción animal: revisión bibliográfica (Revisión)». En: Invest. Agr.: Prod. Sanid. Anim 16.1, 29-60. Online: https: / / bit.ly / 3tR5teg.

Sharma, A. y col. (2017). «Efficient Microorganism compost benefits plant growth and improves soil health in calendula and marigold». En: Horticultural Plant Journal 3.2, 67-72. Online: https: / / bit.ly/3btYdic.

Sosa, B. e Y. García (2019). «Emisión de gases de efecto invernadero en el suelo bajo el uso de abonos verdes». En: Agronomía Mesoamericana 30.3, 767-782. Online: https://bit.ly/3yw9uZs.

Stamberg, Adilson R Paz (2015). «Enfoque sistémico en administración rural: estudio de la unidad 
de producción familiar». En: Ciencias Administrativas 5, 23124-3738. Online: https: / / bit.ly / 3flGB9p.

Steinfeld, H. y col. (2009). La larga sombra del ganado: problemas ambientales y opciones. FAO.

Vargas, A. y col. (2020). Contribuciones académicas a los Diseños y Sostenibilidades en Colombia. Unidad de Publicaciones Universidad de Investigación y Desarrollo - UDI. Online: https : / / bit.ly / 3hrdZyh.
Vezzoli, C. y col. (2014). Product-service system design for sustainability. Routledge. Online: https://bit. ly/33RhENG.

Wang, X. y col. (2016). «Impacts of manure application on soil environment, rainfall use efficiency and crop biomass under dryland farming». En: Scientific reports 6.1, 1-8. Online: https: / / go . nature.com/3omgVNT. 